\title{
The Hausdorff dimension of certain sets arising from Diophantine approximation by restricted sequences of integer vectors
}

by

\author{
BRYAN P. RYNne (Edinburgh)
}

1. Introduction. For positive integers $m, n$, and any positive number $\tau$, define the set $W(m, n ; \tau)$ to be the set of points $X=\left(x_{11}, \ldots, x_{1 n}, \ldots\right.$, $\left.x_{m 1}, \ldots, x_{m n}\right) \in \mathbb{R}^{m n}$ for which there are infinitely many integer vectors $\boldsymbol{q}=\left(q_{1}, \ldots, q_{n}\right) \in \mathbb{Z}^{n}$ such that

$$
\left\|\sum_{j=1}^{n} q_{j} x_{i j}\right\|<|\boldsymbol{q}|^{-\tau}, \quad 1 \leq i \leq m,
$$

where $|\boldsymbol{q}|=\max \left\{\left|q_{j}\right|: j=1, \ldots, n\right\}$, and for any $z \in \mathbb{R},\|z\|$ denotes the distance from $z$ to the nearest integer. The set

$$
W(1,1 ; \tau)=\left\{x \in \mathbb{R}:\|q x\|<|q|^{-\tau} \text { for infinitely many } q \in \mathbb{Z}\right\}
$$

was studied by Jarník [8] and Besicovitch [3] and shown to have Hausdorff dimension $2 /(1+\tau)$, for $\tau>1$. Later Jarník [9] and Eggleston [7] showed that the set

$$
\begin{aligned}
W(m, 1 ; \tau)=\left\{\left(x_{1}, \ldots, x_{m}\right) \in \mathbb{R}^{m}:\left\|q x_{i}\right\|\right. & <|q|^{-\tau}, 1 \leq i \leq m, \\
& \text { for infinitely many } q \in \mathbb{Z}\}
\end{aligned}
$$

has dimension $(m+1) /(1+\tau)$, for $\tau>1 / m$. More recently it was shown by Bovey and Dodson [5] that $\operatorname{dim} W(m, n ; \tau)=m(n-1)+(m+n) /(1+\tau)$, for $\tau>n / m$.

In [7] Eggleston generalized the set $W(m, 1 ; \tau)$ by requiring that the integers $q$ in the definition belong to a given subsequence of integers. To be precise, for any infinite subset $Q \subset \mathbb{Z}^{n}$, let $W_{Q}(m, n ; \tau)$ be the set of points $X \in \mathbb{R}^{m n}$ such that (1.1) holds for infinitely many vectors $\boldsymbol{q} \in Q$. Then, under certain hypotheses on $Q$ (roughly speaking $Q$ must be sufficiently "dense" or sufficiently "sparse") Eggleston obtained the dimension of $W_{Q}(m, 1 ; \tau)$. These results were extended by Borosh and Fraenkel [4], 
who obtained the dimension of this set for all sequences $Q$. In this paper the dimension of $W_{Q}(m, n ; \tau)$ will be determined for all sequences $Q$.

TheOREm. Let $Q \subset \mathbb{Z}^{n}$ be an infinite set of integer vectors and let $\nu$, $0 \leq \nu \leq n$, be the unique number such that the series $\sum_{\boldsymbol{q} \in Q}|\boldsymbol{q}|^{-\nu-\varepsilon}$ is divergent when $\varepsilon<0$, but is convergent when $\varepsilon>0$. Then

$$
\operatorname{dim} W_{Q}(m, n ; \tau)=m(n-1)+(m+\nu) /(1+\tau),
$$

for all $\tau>\nu / m$.

This theorem will be proved in the next section. The result is identical with that in [4] when $n=1$. Also, Bovey and Dodson [5] obtain the dimension of $W_{Q}(m, n ; \tau)$ for certain sequences $Q$ having $\nu=n$ and satisfying a "coprimality" condition which, in essence, ensures that the vectors in the set $Q$ are pairwise linearly independent. No such condition is imposed here, which causes considerable complications in the proof of the Theorem.

We conclude this section by remarking that throughout the above discussion, if $\tau$ does not satisfy the relevant inequality then the corresponding set $W$ or $W_{Q}$ has full Lebesgue measure, i.e. the complement of the set has measure zero. In each case, this follows from Groshev's theorem (see [10]).

2. Proof of Theorem. Since Borosh and Fraenkel have dealt with the case $n=1$, we will assume that $n>1$. Also, for notational simplicity, we will only deal with the case $m=1$. The proof of the general case is similar. We will use the notation $\boldsymbol{x}=\left(x_{1}, \ldots, x_{n}\right)$ for points in $\mathbb{R}^{n}$, and for any $\boldsymbol{x}, \boldsymbol{y} \in \mathbb{R}^{n}$,

$$
\boldsymbol{x} . \boldsymbol{y}=\sum_{j=1}^{n} x_{j} y_{j}, \quad|\boldsymbol{x}|_{2}=(\boldsymbol{x} . \boldsymbol{x})^{1 / 2} .
$$

With this notation we have

$$
W(1, n ; \tau)=\left\{\boldsymbol{x} \in \mathbb{R}^{n}:\|\boldsymbol{q} \cdot \boldsymbol{x}\|<|\boldsymbol{q}|^{-\tau} \text { for infinitely many } \boldsymbol{q} \in Q\right\} .
$$

Before starting the proof we first recall the definition of the Hausdorff dimension of a set $E$ in $\mathbb{R}^{n}$. Let $\mathcal{I}$ be a countable collection of bounded sets $I \subset \mathbb{R}^{n}$. For any $\varrho>0$, the $\varrho$-volume of the collection $\mathcal{I}$ is defined to be

$$
V_{\varrho}(\mathcal{I})=\sum_{I \in \mathcal{I}} d(I)^{\varrho}
$$

where $d(I)=\sup \left\{|\boldsymbol{x}-\boldsymbol{y}|_{2}: \boldsymbol{x}, \boldsymbol{y} \in I\right\}$ is the diameter of $I$. For every $\eta>0$ define

$$
m_{\varrho}(\eta, E)=\inf V_{\varrho}(\mathcal{I}),
$$

where the infimum is taken over all countable collections, $\mathcal{I}$, of sets $I$ with diameter $d(I) \leq \eta$ that cover $E$. Now define the $\varrho$-dimensional Hausdorff 
outer measure of $E$ to be

$$
m_{\varrho}(E)=\sup _{\eta>0} m_{\varrho}(\eta, E) .
$$

The Hausdorff dimension of $E$ is defined to be

$$
\operatorname{dim} E=\inf \left\{\varrho: m_{\varrho}(E)=0\right\} .
$$

We also require some further notation. For any finite set $A$, we let $|A|$ denote the cardinality of $A$. The notation $a \ll b$ (respectively $a \gg b$ ) will denote an inequality of the form $a \leq \alpha b$ (respectively $a \geq \alpha b$ ), where $\alpha>0$ depends at most on $n, \nu$ and certain constants $\gamma, \delta$ and $\varepsilon$ which will be introduced below. If both the inequalities $a \ll b, a \gg b$ hold then we write $a \approx b$. An integral plane in $\mathbb{R}^{n}$ is a set of the form

$$
H(\boldsymbol{q}, t)=\left\{\boldsymbol{x} \in \mathbb{R}^{n}: \boldsymbol{q} \cdot \boldsymbol{x}+t=0\right\}, \quad \boldsymbol{q} \in \mathbb{Z}^{n}, \boldsymbol{q} \neq 0, t \in \mathbb{Z} .
$$

Clearly this is an $(n-1)$-dimensional plane in $\mathbb{R}^{n}$, orthogonal to the integer vector $\boldsymbol{q}$. For any two parallel planes $H, H^{\prime}$, we let $\left|H-H^{\prime}\right|_{2}$ denote the Euclidean distance between the planes, measured along the normal direction. A set $B \subset \mathbb{R}^{n}$ of the form $B=\left\{\boldsymbol{x} \in \mathbb{R}^{n}:|\boldsymbol{x}-\boldsymbol{b}|_{2} \leq d / 2\right\}$ is said to be a ball of diameter $d$ and centre $\boldsymbol{b}$. If $\alpha>0$ is a real number then $\alpha B$ will denote the ball with centre $\boldsymbol{b}$ and diameter $\alpha d$. The unit cube $U$ in $\mathbb{R}^{n}$ is the set

$$
U=\left\{\boldsymbol{x} \in \mathbb{R}^{n}: 0 \leq x_{i} \leq 1, i=1, \ldots, n\right\} .
$$

We can now begin the proof. Suppose that $\tau>\nu$. We first show that

$$
\operatorname{dim} W_{Q}(1, n ; \tau) \leq n-1+(1+\nu) /(1+\tau) .
$$

Since the set $W_{Q}(1, n ; \tau)$ is invariant under translations by integer vectors, it suffices to show that

$$
\operatorname{dim} W_{Q}(1, n ; \tau) \cap U \leq n-1+(1+\nu) /(1+\tau) .
$$

For any non-zero $\boldsymbol{q} \in Q$ and any $t \in \mathbb{Z}$ let

$$
T(\boldsymbol{q}, t)=\left\{\boldsymbol{x} \in U:|\boldsymbol{q} \cdot \boldsymbol{x}+t|<|\boldsymbol{q}|^{-\tau}\right\} .
$$

This set consists of the set of points in $U$ lying within a distance $|\boldsymbol{q}|^{-\tau}|\boldsymbol{q}|_{2}^{-1} \leq$ $|\boldsymbol{q}|^{-(1+\tau)}$ of the integral plane $H(\boldsymbol{q}, t)$. Clearly the number of integers $t$ for which $T(\boldsymbol{q}, t)$ is non-empty is $\ll|\boldsymbol{q}|$. Since $H(\boldsymbol{q}, t)$ is $(n-1)$-dimensional, it can be seen that the set $T(\boldsymbol{q}, t)$ can be covered by a collection $\mathcal{B}(\boldsymbol{q}, t)$ of balls $B$ of diameter $4|\boldsymbol{q}|^{-(1+\tau)}$ such that

$$
|\mathcal{B}(\boldsymbol{q}, t)| \ll|\boldsymbol{q}|^{(1+\tau)(n-1)}
$$

(if $T(\boldsymbol{q}, t)$ is empty we suppose that $\mathcal{B}(\boldsymbol{q}, t)$ is empty). Now, by definition,

$$
W_{Q}(1, n ; \tau) \cap U \subset \bigcup_{\substack{\boldsymbol{q} \in Q \\|\boldsymbol{q}| \geq M}} \bigcup_{t \in \mathbb{Z}} \mathcal{B}(\boldsymbol{q}, t)
$$


for all integers $M \geq 1$, so the set $W_{Q}(1, n ; \tau) \cap U$ is covered by the collection of balls

$$
\mathcal{B}_{M}=\bigcup_{\substack{\boldsymbol{q} \in Q \\|\boldsymbol{q}| \geq M}} \bigcup_{t \in \mathbb{Z}} \mathcal{B}(\boldsymbol{q}, t) .
$$

Now let $\varrho=n-1+(1+\nu) /(1+\tau)+\varepsilon$ for arbitrary $\varepsilon>0$. The $\varrho$-volume of the collection $\mathcal{B}_{M}$ satisfies

$$
\begin{aligned}
V_{\varrho}\left(\mathcal{B}_{M}\right) & \ll \sum_{\substack{\boldsymbol{q} \in Q \\
|\boldsymbol{q}| \geq M}} \sum_{t \in \mathbb{Z}}|\mathcal{B}(\boldsymbol{q}, t)||\boldsymbol{q}|^{-\varrho(1+\tau)} \\
& \ll \sum_{\substack{\boldsymbol{q} \in Q \\
|\boldsymbol{q}| \geq M}}|\boldsymbol{q}|^{1+(1+\tau)(n-1)-\varrho(1+\tau)}=\sum_{\substack{\boldsymbol{q} \in Q \\
|\boldsymbol{q}| \geq M}}|\boldsymbol{q}|^{-\nu-\varepsilon(1+\tau)} .
\end{aligned}
$$

This series is convergent, by the definition of $\nu$, so by taking $M$ sufficiently large the $\varrho$-volume $V_{\varrho}\left(\mathcal{B}_{M}\right)$ can be made arbitrarily small. By the definition of Hausdorff dimension this proves that $\operatorname{dim} W_{Q}(1, n ; \tau) \cap U \leq \varrho$, and since $\varepsilon$ is arbitrarily small, (2.1) follows.

To prove the Theorem we will now prove the reverse inequality for $\operatorname{dim} W_{Q}(1, n ; \tau)$. Suppose, for now, that $\nu>0$ and let $\delta>0$ be an arbitrarily small number satisfying

$$
0<\delta<\min \{\nu, \tau-\nu, 1\} .
$$

Some other restrictions will be imposed on $\delta$ below, but essentially $\delta$ is a fixed "sufficiently small" number.

We also suppose that the series $\sum_{\boldsymbol{q} \in Q}|\boldsymbol{q}|^{-\nu}$ is divergent, i.e. the series in the statement of the theorem is divergent when $\varepsilon=0$. If this assumption does not hold we replace $\nu$ with $\nu+\varepsilon, \varepsilon<0$, throughout the following argument to obtain $\operatorname{dim} W_{Q}(1, n ; \tau) \geq n-1+(1+\nu+\varepsilon) /(1+\tau)$, which yields the result since $\varepsilon<0$ is arbitrary.

Lemma 2.1. For any integer $k_{0}>0$ there exists an integer $k>k_{0}$ such that

$$
\sum_{\substack{\boldsymbol{q} \in Q \\ 2^{k} \leq|\boldsymbol{q}|<2^{k+1}}} 1 \geq 2^{k \nu} / k^{2} .
$$

Proof. The proof is the same as the proof of Lemma 2 of [4].

From now on, $N$ will always denote an integer of the form $2^{k}$, where $k$ is such that (2.2) holds. By Lemma 2.1 there are infinitely many such integers. Thus, writing

$$
Q(N)=\{\boldsymbol{q} \in Q: N \leq|\boldsymbol{q}|<2 N\},
$$


we have

$$
|Q(N)| \geq N^{\nu-\delta / 2}
$$

for all sufficiently large $N$ (of the above form). Now, for any vector $\boldsymbol{q} \in$ $Q(N)$, let $[\boldsymbol{q}] \subset Q$ denote the set of all those vectors $\boldsymbol{q}^{\prime} \in Q(N)$ which are linearly dependent on $\boldsymbol{q}$. Clearly the relation of linear dependence is an equivalence relation on the set $Q(N)$ and we let $[Q(N)]$ denote the corresponding set of equivalence classes $[\boldsymbol{q}]$.

Lemma 2.2. There exists a number $\gamma, \delta \leq \gamma \leq \nu$, and a subset $\widetilde{Q} \subset Q$ such that, for infinitely many $N$,

$$
\begin{aligned}
|[\widetilde{Q}(N)]| & \approx N^{\gamma-\delta}, \\
|[\boldsymbol{q}]| & \approx N^{\nu-\gamma},
\end{aligned}
$$

for all equivalence classes $[\boldsymbol{q}] \in[\widetilde{Q}(N)]$. Thus

$$
|\widetilde{Q}(N)| \approx N^{\nu-\delta} .
$$

Proof. Let $\delta^{\prime}=\delta / 2, t=\left[1 / \delta^{\prime}\right]$ (where [.] denotes the integer part of a number here). For $i=0,1, \ldots, t$, let

$$
\Gamma_{i}(N)=\left\{[\boldsymbol{q}] \in[Q(N)]: N^{i \delta^{\prime}} \leq|[\boldsymbol{q}]|<N^{(i+1) \delta^{\prime}}\right\} .
$$

For all sufficiently large $N$, we must have $|[\boldsymbol{q}]|<2 N<N^{(t+1) \delta^{\prime}}$ so

$$
\sum_{i=0}^{t}\left|\Gamma_{i}(N)\right| N^{(i+1) \delta^{\prime}} \geq|Q(N)| .
$$

Hence, by (2.3) we have, for all sufficiently large $N$,

$$
\sum_{i=0}^{t}\left|\Gamma_{i}(N)\right| N^{(i+2) \delta^{\prime}-\nu} \geq 1 .
$$

Therefore, for some $i$ there exist infinitely many $N$ 's for which

$$
\left|\Gamma_{i}(N)\right| \gg N^{\nu-(i+2) \delta^{\prime}} \text {. }
$$

If $\nu-i \delta^{\prime}>\delta$, put $\gamma=\nu-i \delta^{\prime} \leq \nu$, otherwise put $\gamma=\delta$. Since $\left|\Gamma_{i}(N)\right| \geq 1$ for each of these $N$ 's, we have

$$
\left|\Gamma_{i}(N)\right| \gg N^{\gamma-\delta}, \quad|[\boldsymbol{q}]| \gg N^{i \delta^{\prime}} \geq N^{\nu-\gamma},
$$

for all $[\boldsymbol{q}] \in \Gamma_{i}(N)$. We now select a subset $[Q(N)]^{\prime} \subset \Gamma_{i}(N)$ such that $\left|[Q(N)]^{\prime}\right| \approx N^{\gamma-\delta}$ and for each $[\boldsymbol{q}] \in[Q(N)]^{\prime}$, we select a subclass $[\boldsymbol{q}]^{\prime}$ of the $\boldsymbol{q}$ 's in the equivalence class $[\boldsymbol{q}]$ such that $\left|[\boldsymbol{q}]^{\prime}\right| \approx N^{\nu-\gamma}$. Defining the set $\widetilde{Q}$ to be the union of all the vectors $\boldsymbol{q} \in[\boldsymbol{q}]^{\prime},[\boldsymbol{q}]^{\prime} \in[Q(N)]^{\prime}$, for all the above $N$ 's, it is clear that this set has the properties (2.4), (2.5) and (2.6).

We now suppose that $\nu-\gamma>0$. The case where this does not hold will be discussed below. 
Lemma 2.3. Let $L, \varepsilon>0$ be positive numbers with $L<1$. There exist arbitrarily large integers $N$ such that, for every ball $C \subset U$ with diameter $L$ and every equivalence class $[\boldsymbol{q}] \in[\widetilde{Q}(N)]$ there is a set $S=S(C,[\boldsymbol{q}])$, consisting of pairs $(\boldsymbol{q}, t)$, where $\boldsymbol{q} \in[\boldsymbol{q}]$ and $t \in \mathbb{Z}$, with the properties:

(i) for all $(\boldsymbol{q}, t) \in S$, the plane $H(\boldsymbol{q}, t)$ intersects the ball $\frac{1}{2} C$;

(ii) for all distinct pairs $\left(\boldsymbol{q}^{1}, t^{1}\right),\left(\boldsymbol{q}^{2}, t^{2}\right) \in S$,

$$
\left|H\left(\boldsymbol{q}^{1}, t^{1}\right)-H\left(\boldsymbol{q}^{2}, t^{2}\right)\right|_{2} \gg N^{-1-\nu+\gamma-\delta} ;
$$

(iii) the number of pairs $(\boldsymbol{q}, t)$ in $S$ satisfies

$$
|S| \gg L \chi([\boldsymbol{q}]) \gg L N^{1+\nu-\gamma-\varepsilon},
$$

where $\chi([\boldsymbol{q}])=\sum_{\boldsymbol{q} \in[\boldsymbol{q}]} \phi(|\boldsymbol{q}|)$ and $\phi$ is the Euler function;

(iv) for any set $I \subset C$ with $d(I)>N^{-1+\delta}$,

$$
\left|S_{I}\right| \ll d(I) \chi([\boldsymbol{q}]) \ll d(I) N^{-1+\nu-\gamma},
$$

where $S_{I}$ denotes the set of pairs $(\boldsymbol{q}, t) \in S$ for which $H(\boldsymbol{q}, t)$ intersects $I$.

Proof. To prove the lemma we will reduce the construction, for each equivalence class, to a one-dimensional problem by orthogonally projecting onto the line spanned by the equivalence class and then using the results of [4]. Choose $N$ sufficiently large that it satisfies the conditions imposed on the number $Q$ in the first two sentences of the proof of Lemma 4 of [4]. For any equivalence class $[\boldsymbol{q}] \in[\widetilde{Q}(N)]$ choose some $\boldsymbol{q} \in[\boldsymbol{q}]$ and let $\boldsymbol{u}=\boldsymbol{q} /|\boldsymbol{q}|$ (note that $\boldsymbol{u}$ depends only on $[\boldsymbol{q}]$, not on the particular choice of $\boldsymbol{q} \in[\boldsymbol{q}]$ ). Let $J$ be the interval $[\boldsymbol{c} . \boldsymbol{u}-L / 4, \boldsymbol{c} . \boldsymbol{u}+L / 4]$, where $\boldsymbol{c}$ is the centre of $C$. Now, suppose that the pair of integers $(q, t)$ is such that $q=|\boldsymbol{q}|$ for some $\boldsymbol{q} \in[\boldsymbol{q}]$ and $-t / q \in J$. Then the plane $H(\boldsymbol{q}, t)$ intersects the ball $\frac{1}{2} C$. Also, if $\left(q^{\prime}, t^{\prime}\right)$ is another such pair then the distance between the corresponding integral planes is equal to $\left|t / q-t^{\prime} / q^{\prime}\right|$. Thus, the proof reduces to finding a set of such pairs $(q, t)$ satisfying the analogues of the conditions (ii), (iii) and (iv) (in the analogue of (iv), $I$ becomes a subinterval of $J$ ). However, Lemma 4 of [4] constructs a set of pairs $(q, t)$ having the required properties, and the above condition on the size of $N$ is sufficient to ensure that this construction works for each equivalence class $[\boldsymbol{q}] \in[\widetilde{Q}(N)]$ (note that the notation in $[4]$ is slightly different from ours; in particular, our $\nu-\gamma$ corresponds to $\nu$ in [4]). Thus we have proved the lemma.

We now suppose that $L$ and $C$ are fixed, and choose $N$ so that Lemma 2.3 holds. For any equivalence class $[\boldsymbol{q}] \in[\widetilde{Q}(N)]$ let

$$
E([\boldsymbol{q}])=\bigcup_{(\boldsymbol{q}, t) \in S([\boldsymbol{q}])}(H(\boldsymbol{q}, t) \cap C),
$$

where $S([\boldsymbol{q}])$ is the set constructed in Lemma 2.3 (to simplify the notation slightly we have suppressed the dependence of $S$ on $C$ ). Since the planes 
$H(\boldsymbol{q}, t),(\boldsymbol{q}, t) \in S([\boldsymbol{q}])$, pass through the ball $\frac{1}{2} C$, the $(n-1)$-dimensional Lebesgue measure (which we denote by $\mu_{n-1}$ ) of the set $H(\boldsymbol{q}, t) \cap C$ satisfies $\mu_{n-1}(H(\boldsymbol{q}, t) \cap C) \gg L^{n-1}$, and hence by $(2.8)$,

$$
\mu_{n-1}(E([\boldsymbol{q}])) \gg L^{n} \chi([\boldsymbol{q}]) \gg L^{n} N^{1+\nu-\gamma-\delta}
$$

(taking $\varepsilon<\delta$ ).

Now, for any $\boldsymbol{p} \in \widetilde{Q}(N), \boldsymbol{p} \notin[\boldsymbol{q}], s \in \mathbb{Z}$ and any pair $(\boldsymbol{q}, t) \in S([\boldsymbol{q}])$, let

$$
F(\boldsymbol{p}, s ; \boldsymbol{q}, t)=\left\{\boldsymbol{x} \in H(\boldsymbol{q}, t) \cap C:|\boldsymbol{p} . \boldsymbol{x}+s|<2 n N^{-\nu-\delta}\right\} .
$$

This set consists of the intersection, in $C$, of the hyperplane $H(\boldsymbol{q}, t)$ with the set of points in $\mathbb{R}^{n}$ lying within a distance $2 n N^{-\nu-\delta}|\boldsymbol{p}|_{2}^{-1} \geq N^{-1-\nu-\delta}$ of the hyperplane $H(\boldsymbol{p}, s)$. Thus, since $\boldsymbol{p}$ and $\boldsymbol{q}$ are linearly independent, $F(\boldsymbol{p}, s ; \boldsymbol{q}, t)$ is a (possibly empty) strip on the planar set $H(\boldsymbol{q}, t) \cap C$. Let

$$
F([\boldsymbol{q}])=\bigcup_{\substack{\boldsymbol{p} \in \tilde{Q}(N) \\ \boldsymbol{p} \notin[\boldsymbol{q}]}} \bigcup_{s \in \mathbb{Z}} \bigcup_{(\boldsymbol{q}, t) \in S([\boldsymbol{q}])} F(\boldsymbol{p}, s ; \boldsymbol{q}, t) .
$$

Lemma 2.4. For any $[\boldsymbol{q}] \in[\widetilde{Q}(N)]$,

$$
\frac{\mu_{n-1}(F([\boldsymbol{q}]))}{\mu_{n-1}(E([\boldsymbol{q}]))} \ll L^{-n} N^{-\delta} .
$$

Proof. For any $\boldsymbol{p} \neq 0$ and any $\eta \geq 0$, let

$$
A_{\boldsymbol{p}}(\eta)=\{\boldsymbol{x} \in U:\|\boldsymbol{p} \cdot \boldsymbol{x}\| \leq \eta\} .
$$

It is shown in [10] or in [6] that if $\boldsymbol{p}$ and $\boldsymbol{p}^{\prime}$ are any linearly independent integer vectors then, for any $\eta, \eta^{\prime}>0$,

$$
\mu_{n}\left(A_{\boldsymbol{p}}(\eta) \cap A_{\boldsymbol{p}^{\prime}}\left(\eta^{\prime}\right)\right)=\eta \eta^{\prime}
$$

(the arguments in [6] are geometrical and include pictures which illuminate some of the geometrical arguments used in this part of this paper). Now, by definition,

so

$$
F([\boldsymbol{q}]) \subset \bigcup_{\substack{\boldsymbol{p} \in \tilde{Q}(N) \\ \boldsymbol{p} \notin[\boldsymbol{q}]}} \bigcup_{\boldsymbol{q} \in[\boldsymbol{q}]}\left(A_{\boldsymbol{p}}\left(2 n N^{-\nu-\delta}\right) \cap A_{\boldsymbol{q}}(0)\right),
$$

$$
\mu_{n-1}(F([\boldsymbol{q}])) \leq \sum_{\substack{\boldsymbol{p} \in \tilde{Q}(N) \\ \boldsymbol{p} \notin[\boldsymbol{q}]}} \sum_{\boldsymbol{q} \in[\boldsymbol{q}]} \mu_{n-1}\left(A_{\boldsymbol{p}}\left(2 n N^{-\nu-\delta}\right) \cap A_{\boldsymbol{q}}(0)\right) .
$$

For $\eta>0$ the set $A_{\boldsymbol{p}}\left(2 n N^{-\nu-\delta}\right) \cap A_{\boldsymbol{q}}(\eta)$ is an $n$-dimensional "thickening" of the set $A_{\boldsymbol{p}}\left(2 n N^{-\nu-\delta}\right) \cap A_{\boldsymbol{q}}(0)$ (which consists of portions of $(n-1)$ dimensional planes) with "thickness" $\eta|\boldsymbol{q}|_{2}^{-1}$. Thus

$$
\mu_{n-1}\left(A_{\boldsymbol{p}}\left(2 n N^{-\nu-\delta}\right) \cap A_{\boldsymbol{q}}(0)\right)=\lim _{\eta \rightarrow 0} \mu_{n}\left(A_{\boldsymbol{p}}\left(2 n N^{-\nu-\delta}\right) \cap A_{\boldsymbol{q}}(\eta)\right) / \eta|\boldsymbol{q}|_{2}^{-1} .
$$


Hence by (2.5), (2.6), (2.11) and (2.12),

$$
\begin{aligned}
\mu_{n-1}(F([\boldsymbol{q}])) & \leq \sum_{\substack{\boldsymbol{p} \in \tilde{Q}(N) \\
\boldsymbol{p} \notin[\boldsymbol{q}]}} \sum_{\boldsymbol{q} \in[\boldsymbol{q}]} \lim _{\eta \rightarrow 0} 2 n N^{-\nu-\delta} \eta / \eta|\boldsymbol{q}|_{2}^{-1} \\
& \ll N^{\nu-\delta} N^{\nu-\gamma} N^{1-\nu-\delta}=N^{1+\nu-\gamma-2 \delta},
\end{aligned}
$$

so the result follows from (2.10).

Now, by geometrical arguments, it can be seen from Lemma 2.4 and the construction of the set $F([\boldsymbol{q}])$ that for $N$ sufficiently large we can choose a collection $\mathcal{B}([\boldsymbol{q}])$ of pairwise disjoint balls $B \subset C$, of diameter $n^{-1}(2 N)^{-(1+\tau)}$, whose centres $\boldsymbol{b}$ lie on $E([\boldsymbol{q}]) \backslash F([\boldsymbol{q}])$, and satisfy

$$
\left|\boldsymbol{b}-\boldsymbol{b}^{\prime}\right|_{2} \geq N^{-(1+\tau)} \quad \text { if } \boldsymbol{b} \neq \boldsymbol{b}^{\prime}
$$

and such that

$$
|\mathcal{B}([\boldsymbol{q}])| \gg \mu_{n-1}(E([\boldsymbol{q}])) /\left(N^{-(\tau+1)}\right)^{n-1} \gg L^{n} \chi([\boldsymbol{q}]) N^{(1+\tau)(n-1)}
$$

(by $(2.10)$ ).

Repeating the above constructions for all $[\boldsymbol{q}] \in[\widetilde{Q}(N)]$ we can define the collection of balls

$$
\mathcal{B}=\bigcup_{[\boldsymbol{q}] \in[\tilde{Q}(N)]} \mathcal{B}([\boldsymbol{q}])
$$

If $B \in \mathcal{B}([\boldsymbol{q}]), B^{\prime} \in \mathcal{B}\left([\boldsymbol{q}]^{\prime}\right),[\boldsymbol{q}] \neq[\boldsymbol{q}]^{\prime}$, and $\boldsymbol{b}, \boldsymbol{b}^{\prime}$ are the centres of $B, B^{\prime}$, respectively, then it follows from the definition of the sets $F(\boldsymbol{p}, s ; \boldsymbol{q}, t)$ and the fact that $\boldsymbol{b} \in E([\boldsymbol{q}]) \backslash F([\boldsymbol{q}])$ that

$$
\left|\boldsymbol{b}-\boldsymbol{b}^{\prime}\right|_{2} \geq N^{-1-\nu-\delta} .
$$

Collecting together the above results we obtain the following lemma.

Lemma 2.5. Let $L, \varepsilon>0$ be positive numbers with $L<1$. There exist arbitrarily large integers $N$ such that for every ball $C \subset U$ with diameter $L$ there is a collection $\mathcal{B}$ of pairwise disjoint balls $B \subset C$, such that:

(i) each $B \in \mathcal{B}$ has diameter $n^{-1}(2 N)^{-(1+\tau)}$;

(ii) for all $B \in \mathcal{B}$, there exists $\boldsymbol{q} \in \widetilde{Q}(N)$ such that

$$
\|\boldsymbol{q} \cdot \boldsymbol{x}\|<|\boldsymbol{q}|^{-\tau} \quad \text { for all } \boldsymbol{x} \in B
$$

(iii) $|\mathcal{B}| \geq c_{1} L^{n} X(N) N^{(1+\tau)(n-1)}$, where $X(N)=\sum_{[\boldsymbol{q}] \in[\tilde{Q}(N)]} \chi([\boldsymbol{q}]) \gg$ $N^{1+\nu-\delta-\varepsilon}$.

Proof. Since by construction the balls in the collections $\mathcal{B}([\boldsymbol{q}])$ have diameter $n^{-1}(2 N)^{-(1+\tau)}$, (i) holds. The fact that the balls in $\mathcal{B}$ are pairwise disjoint follows from (i) and (2.13) and (2.15) for $N$ sufficiently large (since 
$\tau>\nu+\delta)$. Now, for any $B \in \mathcal{B}([\boldsymbol{q}])$, and any $\boldsymbol{x} \in B$, there is a pair $(\boldsymbol{q}, t) \in S([\boldsymbol{q}])$ such that the centre $\boldsymbol{b}$ of $B$ lies on $H(\boldsymbol{q}, t)$, and hence

$$
\begin{aligned}
|\boldsymbol{q} \cdot \boldsymbol{x}+t| & \leq|\boldsymbol{q} \cdot \boldsymbol{b}+t|+|\boldsymbol{q} \cdot(\boldsymbol{x}-\boldsymbol{b})| \\
& \leq|\boldsymbol{q}|_{2}|\boldsymbol{x}-\boldsymbol{b}|_{2} \leq n^{1 / 2}|\boldsymbol{q}| n^{-1}(2 N)^{-(1+\tau)}<|\boldsymbol{q}|^{-\tau},
\end{aligned}
$$

which shows that (ii) holds for all $B \in \mathcal{B}([\boldsymbol{q}])$, and hence for all $B \in \mathcal{B}$. To show that (iii) holds we combine the estimate (2.14) with the definition of $\mathcal{B}$ to yield

$$
|\mathcal{B}| \gg \sum_{[\boldsymbol{q}] \in[\tilde{Q}(N)]} L^{n} \chi([\boldsymbol{q}]) N^{(1+\tau)(n-1)}=L^{n} X(N) N^{(1+\tau)(n-1)} .
$$

The estimate for $X(N)$ follows from (2.4) and (2.8). This completes the proof.

LEMmA 2.6. Let $\mathcal{B}$ be the collection of balls constructed in Lemma 2.5 and let $I$ be a set in $\mathbb{R}^{n}$ with $d(I) \geq \frac{1}{2} n^{-1}(2 N)^{-(1+\tau)}$, which intersects $h$ of the balls $B$ in $\mathcal{B}$. Then if $d(I) \leq N^{-1+\delta}$,

$$
h \leq c_{2} d(I)^{n-1} N^{(1+\tau)(n-1)}\left(1+d(I) N^{1+\nu+\delta}\right),
$$

while if $d(I)>N^{-1+\delta}$,

$$
h \leq c_{2} d(I)^{n} N^{(1+\tau)(n-1)} X(N) .
$$

Proof. For any $[\boldsymbol{q}] \in[\widetilde{Q}(N)]$ and any pair $(\boldsymbol{q}, t) \in S([\boldsymbol{q}])$, let $\mathcal{B}(\boldsymbol{q}, t)$ be the set of balls $B \in \mathcal{B}$ whose centres lie on the plane $H(\boldsymbol{q}, t)$. We begin by estimating the number $h(\boldsymbol{q}, t)$ of balls $B \in \mathcal{B}(\boldsymbol{q}, t)$ which can intersect $I$. Since the balls $B \in \mathcal{B}(\boldsymbol{q}, t)$ have diameters $n^{-1}(2 N)^{-(1+\tau)}$ and their centres all lie on the $(n-1)$-dimensional plane $H(\boldsymbol{q}, t)$ and are a distance at least $N^{-(1+\tau)}$ apart, it follows from volume considerations on the plane $H(\boldsymbol{q}, t)$ that

$$
h(\boldsymbol{q}, t) \ll\left(\frac{d(I)}{N^{-(1+\tau)}}\right)^{n-1} .
$$

Now suppose that $d(I) \leq N^{-1-\nu-\delta} / 4$. In this case it follows from the construction of the collection $\mathcal{B}$ that $I$ can intersect balls from at most $\ll 1$ collections $\mathcal{B}(\boldsymbol{q}, t)$, thus $h \ll h(\boldsymbol{q}, t)$. Next, suppose that $N^{-1-\nu-\delta} / 4<$ $d(I) \leq N^{-1+\delta}$. Then we can choose a collection of balls $D$ of diameter $N^{-1-\nu-\delta} / 4$ covering $I$, such that the number $h^{\prime}$ of balls $D$ is bounded by

$$
h^{\prime} \ll\left(\frac{d(I)}{N^{-1-\nu-\delta}}\right)^{n} .
$$

Using (2.18) to estimate the number of balls $B$ intersecting each $D$ we obtain, 
for this case,

$$
h \ll h^{\prime}\left(\frac{N^{-1-\nu-\delta}}{N^{-(1+\tau)}}\right)^{n-1}=d(I)^{n} N^{1+\nu+\delta+(1+\tau)(n-1)} .
$$

Combining (2.18) and (2.19) proves (2.16).

Now, suppose that $d(I)>N^{-1+\delta}$. Then by (iv) of Lemma 2.3, for each $[\boldsymbol{q}] \in[\widetilde{Q}(N)]$, the number $h^{\prime \prime}$ of collections $\mathcal{B}(\boldsymbol{q}, t),(\boldsymbol{q}, t) \in S([\boldsymbol{q}])$, which have at least one ball intersecting the set $I$ is bounded by $h^{\prime \prime} \ll d(I) \chi([\boldsymbol{q}])$. Thus, using (2.18),

$$
h \leq \sum_{[\boldsymbol{q}] \in[\tilde{Q}(N)]} d(I) \chi([\boldsymbol{q}]) d(I)^{n-1} N^{(1+\tau)(n-1)}=d(I)^{n} X(N) N^{(1+\tau)(n-1)},
$$

which completes the proof.

Now let $\varrho=n-1+(1+\nu) /(1+\tau)-\delta$. We will prove that $\operatorname{dim} W_{Q}(1, n ; \tau)$ $\geq \varrho$, which, since $\delta$ is arbitrarily small, proves the Theorem. The proof is based on some of the methods used in [1] and [2]. Choose $N_{0}>0$ sufficiently large that

$$
N_{0}^{1+\nu-(1+\delta)(1+\tau)} \leq c_{1} c_{2}^{-1} 4^{-n-1}
$$

(this is possible since $\tau>\nu$ ). Let $\mathcal{F}$ be any countable family of sets $I$ in $\mathbb{R}^{n}$ of positive diameter $d(I) \leq \frac{1}{2} n^{-1}\left(2 N_{0}\right)^{-(1+\tau)}$ with

$$
V_{\varrho}(\mathcal{F})=\sum_{I \in \mathcal{F}} d(I)^{\varrho}<1 .
$$

We will show that the family $\mathcal{F}$ cannot cover the set $W_{Q}(1, n ; \tau)$ and hence, by definition, $m_{\varrho}\left(W_{Q}(1, n ; \tau)\right)>0$, which proves that $\operatorname{dim} W_{Q}(1, n ; \tau) \geq \varrho$. To do this we construct a sequence of sets $U \supset J_{0} \supset J_{1} \supset \ldots$, where $J_{j}$ is the union of $M_{j}>0$ pairwise disjoint balls and integers $N_{0}<N_{1}<\ldots$ such that for $j \geq 1$, the following conditions are satisfied:

(i) ${ }_{j} J_{j}$ intersects no $I \in \mathcal{F}$ with $d(I)>\frac{1}{2} n^{-1}\left(2 N_{j}\right)^{-(1+\tau)}$;

(ii) ${ }_{j}$ each ball of $J_{j}$ has diameter $n^{-1}\left(2 N_{j}\right)^{-(1+\tau)}$;

(iii) ${ }_{j}$ if $\boldsymbol{x} \in J_{j}$, there is a $\boldsymbol{q} \in \widetilde{Q}\left(N_{j}\right)$ such that $\|\boldsymbol{q} \cdot \boldsymbol{x}\|<|\boldsymbol{q}|^{-\tau}$;

(iv) ${ }_{j} M_{j} \geq 4 c_{2} c_{1}^{-1} n^{n} 2^{n(1+\tau)} N_{j}^{1+\nu-\delta(1+\tau)+(1+\tau)(n-1)}$.

Supposing that such sequences exist, let

$$
J_{\infty}=\bigcap_{j=0}^{\infty} J_{j} .
$$

Since the sequence $J_{j}, j=0,1, \ldots$, is a decreasing sequence of non-empty closed bounded sets in $\mathbb{R}, J_{\infty}$ is non-empty. By $(\mathrm{i})_{j}, J_{\infty}$ does not intersect any set $I \in \mathcal{F}$, while by (iii) ${ }_{j}, J_{\infty} \subset W_{Q}(1, n ; \tau)$. Thus, $\mathcal{F}$ does not cover $W_{Q}(1, n ; \tau)$. 
The construction is by induction. Let $J_{0}$ be the ball of diameter 1 and centre $\left(\frac{1}{2}, \ldots, \frac{1}{2}\right)$, and let $N_{0}$ be as above. Now suppose that $J_{0}, J_{1}, \ldots, J_{j-1}$, $N_{0}, N_{1}, \ldots, N_{j-1}$ have already been constructed satisfying the above conditions, for some $j \geq 1$. We will construct $J_{j}$ and $N_{j}$. Let $D$ be a ball of $J_{j-1}$ and let $C=\frac{1}{4} D$. Applying Lemma 2.5 to $C$ we choose $N_{j}=N$ such that $N_{j}^{-1+\delta}<n^{-1}\left(2 N_{j-1}\right)^{-(1+\tau)}$, and we obtain the corresponding collection of balls $\mathcal{B}=\mathcal{B}(D)$. Let

$$
\mathcal{G}_{j}=\bigcup_{D \in J_{j-1}} \mathcal{B}(D)
$$

Now let

$$
\begin{aligned}
& \mathcal{F}_{j}^{1}=\left\{I \in \mathcal{F}: \frac{1}{2} n^{-1}\left(2 N_{j}\right)^{-(1+\tau)}<d(I) \leq N_{j}^{-1+\delta}\right\}, \\
& \mathcal{F}_{j}^{2}=\left\{I \in \mathcal{F}: N_{j}^{-1+\delta}<d(I) \leq \frac{1}{2} n^{-1}\left(2 N_{j-1}\right)^{-(1+\tau)}\right\},
\end{aligned}
$$

and let $\mathcal{H}_{j}$ be the set of balls in $\mathcal{G}_{j}$ which intersect a set $I \in \mathcal{F}_{j}^{1} \cup \mathcal{F}_{j}^{2}$. We define $J_{j}$ to be the union of the balls in the collection $\mathcal{G}_{j} \backslash \mathcal{H}_{j}$. Thus, we have $J_{j} \subset J_{j-1}$ and (i) ${ }_{j}$ holds (because $d(I) \leq \frac{1}{2} n^{-1}\left(2 N_{0}\right)^{-(1+\tau)}, I \in \mathcal{F}$, if $j=1$, and because of (i) $)_{j-1}$ if $j>1$ ). Also, (ii) $)_{j}$ and (iii) $)_{j}$ follow from (i) and (ii) of Lemma 2.5. It remains to consider (iv) ${ }_{j}$.

If $I \in \mathcal{F}_{j}^{1} \cup \mathcal{F}_{j}^{2}, I$ cannot intersect $\mathcal{B}(D)$ for two distinct balls $D \in J_{j-1}$ (because of (ii) $j_{-1}$, if $j>1$ ). Therefore, by Lemma 2.6,

$$
\begin{aligned}
\left|\mathcal{H}_{j}\right| \leq & c_{2} \sum_{I \in \mathcal{F}_{j}^{1}} d(I)^{n-1} N_{j}^{(1+\tau)(n-1)}\left(1+d(I) N_{j}^{1+\nu+\delta}\right) \\
& +c_{2} \sum_{I \in \mathcal{F}_{j}^{2}} d(I)^{n} N_{j}^{(1+\tau)(n-1)} X\left(N_{j}\right) .
\end{aligned}
$$

It follows from $(2.21)$ and the definitions of $\mathcal{F}_{j}^{1}, \mathcal{F}_{j}^{2}$, that, if $\delta$ is sufficiently small,

$$
\begin{gathered}
\sum_{I \in \mathcal{F}_{j}^{1}} d(I)^{n-1} \leq N_{j}^{1+\nu-\delta(1+\tau)}, \quad \sum_{I \in \mathcal{F}_{j}^{1}} d(I)^{n} \leq N_{j}^{-(1-\delta)(1-(1+\nu) /(1+\tau)+\delta)} \\
\sum_{I \in \mathcal{F}_{j}^{2}} d(I)^{n} \leq N_{j-1}^{1+\nu-(1+\delta)(1+\tau)}
\end{gathered}
$$

Hence,

$$
\begin{aligned}
\left|\mathcal{H}_{j}\right| \leq & c_{2}\left(N_{j}^{1+\nu-\delta(1+\tau)}+N_{j}^{1+\nu+\delta-(1-\delta)(1-(1+\nu) /(1+\tau)+\delta)}\right. \\
& \left.+N_{j-1}^{1+\nu-(1+\delta)(1+\tau)} X\left(N_{j}\right)\right) N_{j}^{(1+\tau)(n-1)} \\
\leq & 2 c_{2} N_{j-1}^{1+\nu-(1+\delta)(1+\tau)} X\left(N_{j}\right) N_{j}^{(1+\tau)(n-1)}
\end{aligned}
$$

for sufficiently large $N_{j}$ if $\delta$ is sufficiently small (using the estimate for $X(N)$ in Lemma 2.5 with $\varepsilon$ sufficiently small). 
Now suppose that $j=1$. By (iii) of Lemma 2.5 (with $d(C)=1 / 4$ ), together with (2.20) and (2.23),

$$
\left|\mathcal{G}_{1}\right| \geq c_{1} 4^{-n} X\left(N_{1}\right) N_{1}^{(1+\tau)(n-1)} \geq 2\left|\mathcal{H}_{1}\right| .
$$

Hence

$$
M_{1} \geq\left|\mathcal{G}_{1}\right|-\left|\mathcal{H}_{1}\right| \geq c_{1} 2^{-2 n-1} X\left(N_{1}\right) N_{1}^{(1+\tau)(n-1)},
$$

so (iv) ${ }_{1}$ holds for sufficiently large $N_{1}$ if $\delta$ is sufficiently small (again using the above estimate for $X(N)$ ).

Next suppose that $j>1$. Then, by (iii) of Lemma 2.5 , (ii) $)_{j-1}$ and (iv) $)_{j-1}$

$$
\begin{aligned}
\left|\mathcal{G}_{j}\right| & \geq M_{j-1} c_{1} n^{-n}\left(2 N_{j-1}\right)^{-n(1+\tau)} X\left(N_{j}\right) N_{j}^{(1+\tau)(n-1)} \\
& \geq 4 c_{2} N_{j-1}^{1+\nu-(1+\delta)(1+\tau)} X\left(N_{j}\right) N_{j}^{(1+\tau)(n-1)} \geq 2\left|\mathcal{H}_{j}\right| .
\end{aligned}
$$

Thus, $M_{j} \geq\left|\mathcal{G}_{j}\right|-\left|\mathcal{H}_{j}\right| \geq\left|\mathcal{G}_{j}\right| / 2$, and it follows from (2.24) that (iv) ${ }_{j}$ holds for sufficiently large $N_{j}$ if $\delta$ is sufficiently small. This completes the proof of the Theorem when $\nu-\gamma>0$.

To obtain the lower bound for $\operatorname{dim} W_{Q}(1, n ; \tau)$ in the cases when $\nu-\gamma=$ $0, \nu>0$, and when $\nu=0$, we observe that in both these cases we may choose a subset $\widetilde{Q} \subset Q$ such that for an increasing sequence of $N$ 's, $\widetilde{Q}(N)$ contains exactly one vector $\boldsymbol{q}$. This is the analogue of Lemma 2.2 for this case and provides sufficient vectors to obtain the required lower bound. Since there is only one vector $\boldsymbol{q} \in \widetilde{Q}(N)$ the analogue of Lemma 2.3 can easily be obtained (with $\nu-\gamma=\delta=\varepsilon=0$, and omitting the function $\chi$ ) simply by considering the set of planes $H(\boldsymbol{q}, t), t \in \mathbb{Z}$ which intersect $\frac{1}{2} C$. Similarly, the collection $\mathcal{B}$ of Lemma 2.5 can be constructed easily (the sets $F$ and Lemma 2.4 are not now required since these planes are parallel and hence do not intersect each other) and the analogue of Lemma 2.6 can be proved by the same means as above. Finally, the required lower bound can be obtained as before.

\section{References}

[1] A. Baker and W. M. Schmidt, Diophantine approximation and Hausdorff dimension, Proc. London Math. Soc. 21 (1970), 1-11.

[2] R. C. Baker, Singular n-tuples and Hausdorff dimension, Math. Proc. Cambridge Philos. Soc. 81 (1977), 377-385.

[3] A. S. Besicovitch, Sets of fractional dimensions (IV): On rational approximation to real numbers, J. London Math. Soc. 9 (1934), 126-131.

[4] I. Borosh and A. S. Fraenkel, A generalization of Jarnik's theorem on Diophantine approximations, Indag. Math. 34 (1972), 193-201.

[5] J. D. Bovey and M. M. Dodson, The Hausdorff dimension of systems of linear forms, Acta Arith. 45 (1986), 337-358.

[6] M. M. Dodson and J. A. G. Vickers, Exceptional sets in Kolmogorov-Arnol'dMoser theory, J. Phys. A 19 (1986), 349-374. 
[7] H. G. Eggleston, Sets of fractional dimensions which occur in some problems of number theory, Proc. London Math. Soc. 54 (1951), 42-93.

[8] V. Jarník, Diophantische Approximationen und Hausdorffsches Mass, Mat. Sb. 36 (1929), 371-382.

[9] —, Über die simultanen diophantischen Approximationen, Math. Z. 33 (1931), 505543.

[10] V. G. Sprindžuk, Metric Theory of Diophantine Approximations (translated by R. A. Silverman), John Wiley, 1979.

DEPARTMENT OF MATHEMATICS

HERIOT-WATT UNIVERSITY

RICCARTON

EDINBURGH EH14 4AS

SCOTLAND 